\title{
Successful Treatment of Ventriculitis Caused by MDR/XDR Gram-Negative Bacillus Using Ceftazidime/Avibactam: Case Series and Literature Review
}

\author{
Qian Zhou' \\ Hao Wang ${ }^{2}$ \\ Tianxiang Zhan ${ }^{2}$ \\ Xiaofeng Yang ${ }^{1,2}$ \\ Liang Wen ${ }^{2}$ \\ 'Department of Emergency and Trauma \\ Center, The International Medical \\ Center, The First Affiliated Hospital, \\ Zhejiang University School of Medicine, \\ Hangzhou, Zhejiang Province, 310003, \\ People's Republic of China; ${ }^{2}$ Department \\ of Neurosurgery, The First Affiliated \\ Hospital, Zhejiang University School of \\ Medicine, Hangzhou, Zhejiang Province, \\ 310003, People's Republic of China
}

Correspondence: Liang Wen

Department of Neurosurgery, The First Affiliated Hospital, Zhejiang University School of Medicine, 79 Qingchun Road, Hangzhou, 310003, Zhejiang Province,

People's Republic of China

Email wenliang@zju.edu.cn

Xiaofeng Yang

Department of Emergency and Trauma

Center, The International Medical Center,

The First Affiliated Hospital, Zhejiang

University School of Medicine, 79

Qingchun Road, Hangzhou, 310003,

Zhejiang Province, People's Republic of

China

Email zjcswk@zju.edu.cn
Background: Central nervous system (CNS) infections caused by multidrug-resistant (MDR) and extensively drug-resistant (XDR) Gram-negative bacillus, including carbapenem-resistant Enterobacteriaceae (CRE) and Pseudomonas aeruginosa, are associated with high mortality rates. Clinical trials of ceftazidime/avibactam (CAZ/AVI) on infections of other systems indicate that they are effective against these infections. However, clinical studies on the efficacies of CAZ/AVI in the treatment of CNS infections have not been done. Case Presentation: We evaluated 3 patients diagnosed with MDR/XDR Gram-negative bacillus-associated CNS infections, and effectively treated with CAZ/AVI. Moreover, we performed literature reviews. Before the onset of CNS infections, the 3 patients were subjected to neurosurgical operations, treated with mechanical ventilation, long-term intensive care unit therapy, and various antibiotics. By intravenously administering CAZ/AVI, combined with another antibiotic, the MDR/XDR K. pneumoniae and P. aeruginosa associated ventriculitis was effectively treated in the 3 patients.

Conclusion: CAZ/AVI is a viable treatment option for CNS infections caused by MDR/ XDR Gram-negative bacteria.

Keywords: ceftazidime-avibactam, extensively drug-resistant Gram-negative bacillus, Klebsiella pneumoniae, Pseudomonas aeruginosa, ventriculitis infection

\section{Introduction}

Bacterial infections of the central nervous system (CNS) are associated with high mortality rates and present a substantial global challenge. ${ }^{1}$ Antibiotics are vital in the treatment of bacterial infectious. However, therapeutic options for intracranial infections are increasingly limited by increasing microbial resistance, specifically multidrug-resistant (MDR) and extensively drug-resistant (XDR) Gram-negative bacillus.

Ceftazidime/avibactam (CAZ/AVI), combination antibiotics of cephalosporin and $\beta$-lactamase inhibitors, used in the treatment of complicated urinary tract or intraabdominal infections, have exhibited a potential activity against MDR/XDR Gramnegative bacillus. ${ }^{2}$ However, studies on the efficacies of ceftazidime/avibactam for CNS infections are limited. In this study, we report on 3 patients with postneurosurgical ventriculitis, ie, one with carbapenem-resistant MDR Pseudomonas aeruginosa infection and 2 with carbapenem-resistant XDR Klebsiella pneumoniae infections. These patients were effectively treated with CAZ/AVI. 


\section{Case Presentation}

\section{Case I}

An elderly patient underwent decompressive craniectomy for a right traumatic intracerebral hematoma and subsequent tracheotomy at a local hospital. After one month, he was transferred to a rehabilitation facility. During treatment in the rehabilitation facility, he developed a cerebrospinal fluid (CSF) incision leakage, followed by persistent fever and progressive consciousness disturbance. The CSF test of the patient revealed significant leukocytosis and decreased glucose levels, indicating a CNS infection. The CSF smear confirmed the presence of Gram-negative bacteria, consequently, he was immediately sent to our hospital for further treatment. Upon admission, physical examination revealed a poorly healed scalp incision with pus exudates from the subcutaneous tissue. Enhanced computed tomography (CT) scan of the brain identified the right subcutaneous abscess (Figure 1A and B) and possible pus of longitudinal cistern (Figure 1C). Emergency debridement and extra-ventricular drainage (EVD) was performed. During the procedure, substantial amounts of pus were observed in the subcutaneous tissue and the former intracerebral hematoma cavity penetrated to the lateral ventricle (Supplementary video 1). The pus, necrotic tissue, and artificial meninges were completely removed, after which the fascia of the patient was used to repair defective meninges. After operation, the patient was intravenously (IV) treated with $2 \mathrm{~g}$ of meropenem at intervals of every 8 hours based on CSF tests of a local hospital. On the 6th day of hospitalization, CSF culture revealed Klebsiella pneumoniae carbapenemase (KPC)-producing XDR $K$. pneumoniae. Susceptibility tests showed that CAZ/AVI is an effective therapeutic option that exhibited synergistic effects with meropenem (Table 1). The anti-infectious therapy was changed to 2 $\mathrm{g}$ of meropenem combined with $2.5 \mathrm{~g}$ of CAZ/AVI IV every 8 hours. Thereafter, leukocyte counts in the CSF gradually decreased with increasing glucose levels; however, a recurrence of the abnormal tests (Table 2) and fever were observed. On the 8th day of hospitalization, EVD was removed and continuous lumbar cistern CSF drainage was performed on the 10th day of hospitalization. On the 19th day of hospitalization, the CSF culture was first negative. On the 21st day of hospitalization, with apparent improvement of CSF traits and tests, the drainage tube of the lumbar cistern was removed. The CSF white blood cell (WBC) count was low; then, CSF glucose levels increased in subsequent CSF tests. After repeated negative results upon CSF culture, the antibiotics (CAZ/AVI plus meropenem) were administered until the 32nd day of hospitalization. Then, the patient was transferred to a rehabilitation facility. The CSF test and susceptibility results for XDR K. pneumoniae during his hospital stay are shown in Tables 1 and 2, respectively. A summary of the clinical treatment process of Case 1 is shown in Figure 2.

\section{Case 2}

A 53-year-old male underwent decompressive craniectomy with intracranial hematoma removal, and simultaneous bilateral EVD for a cerebral hemorrhage combined with obstructive hydrocephalus at a local hospital. After the operation, the patient presented with fever, and a CSF culture revealed XDR K. pneumoniae. He was transferred to our hospital. Based on the findings of the CSF culture, the CNS infection was treated with 500,000 units of polymyxin B and $100 \mathrm{mg}$ of tigecycline IV at $12 \mathrm{~h}$ intervals, combined with intracerebroventricular injection of 50,000 units of polymyxin B every day. On the 4th day of hospitalization, the CSF culture of the patient in our institution was confirmed as MDR K. pneumoniae infection (Table 3) while the result of sputum culture was MDR Pseudomonas aeruginosa (Table 4). Both bacteria were sensitive to polymyxin; therefore, the original antibiotic regimen was continued. Leukocyte counts in the CSF gradually decreased with increasing glucose levels (Table 5); however,
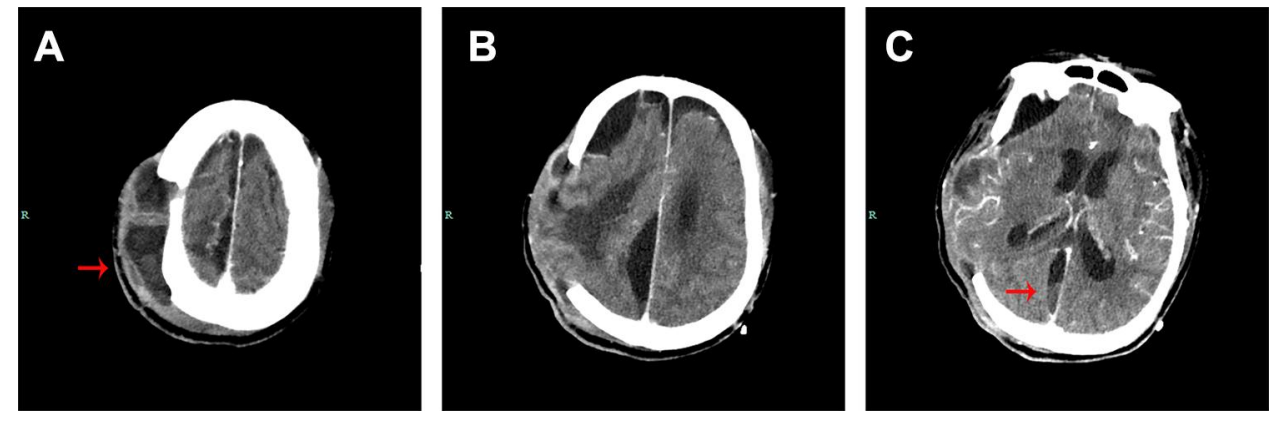

Figure I Brain computed tomography scan of case I. (A and B) CT images show pus gathering in the subcutaneous tissues. (C) CT images show the pus gathering in the cisterns of interhemispheric fissure (red arrows). 
Table I Susceptibility Results for Klebsiella pneumoniae in Cerebrospinal Fluid Collected on Different Date in case I

\begin{tabular}{|c|c|c|c|c|c|}
\hline \multicolumn{2}{|c|}{ Day 3} & \multicolumn{2}{|c|}{ Day 4 and 6} & \multicolumn{2}{|c|}{ Day I3 } \\
\hline Antibiotic & MIC (mg/L) & Antibiotic & MIC (mg/L) & Antibiotic & MIC (mg/L) \\
\hline Tigecycline & 2 & Tigecycline & 2 & Tigecycline & 2 \\
\hline Ceftazidime/avibactam & $21 \mathrm{~mm} *$ & Ceftazidime/avibactam & $21 \mathrm{~mm} *$ & Ceftazidime/averbatan & $21 \mathrm{~mm} *$ \\
\hline Levofloxacin & $\geq 8$ & Imipenem & $\geq 16$ & Meropenem & 6 \\
\hline Cefuroxime sodium & $\geq 64$ & Levofloxacin & $\geq 8$ & Ampicillin & 6 \\
\hline Cefuroxime axetil & $\geq 64$ & Minocycline & $\geq 16$ & Aztreonam & 6 \\
\hline $\begin{array}{l}\text { Compound } \\
\text { sulfamethoxazole }\end{array}$ & $\leq 20$ & $\begin{array}{l}\text { Compound } \\
\text { sulfamethoxazole }\end{array}$ & $\leq 20$ & Ciprofloxacin & 6 \\
\hline Ceftazidime & $\geq 64$ & Ceftazidime & $\geq 64$ & Gentamicin & 6 \\
\hline Cefepime & $\geq 32$ & Tobramycin & $\geq 16$ & Cefazolin & 6 \\
\hline $\begin{array}{l}\text { Piperacillin/tazobactam } \\
\text { sodium }\end{array}$ & $\geq 128$ & Cefepime & $\geq 32$ & Cefepime & $\geq 32$ \\
\hline Cefoperazone/sulbactam & $\geq 64$ & $\begin{array}{l}\text { Piperacillin/tazobactam } \\
\text { sodium }\end{array}$ & $\geq 128$ & $\begin{array}{l}\text { Piperacillin/tazobactam } \\
\text { sodium }\end{array}$ & $\geq 128$ \\
\hline Ertapenem & $\geq 8$ & Meropenem & $\geq 16$ & Cefoperazone/sulbactam & $\geq 64$ \\
\hline Imipenem & $\geq 16$ & Cefoperazone/sulbactam & $\geq 64$ & Ertapenem & $\geq 8$ \\
\hline Cefoxitin & $\geq 64$ & Polymyxin & $\leq 0.5$ & Ceftazidime & $\geq 64$ \\
\hline Cefatriaxone & $\geq 64$ & Ciprofloxacin & $\geq 4$ & $\begin{array}{l}\text { Compound } \\
\text { sulfamethoxazole }\end{array}$ & $\leq 20$ \\
\hline Amikacin & $\geq 64$ & Aztreonam & $\geq 64$ & Cefuroxime axetil & $\geq 64$ \\
\hline $\begin{array}{l}\text { Amoxicillin/clavulanate } \\
\text { potassium }\end{array}$ & $\geq 32$ & Amikacin & $\geq 64$ & Levofloxacin & $\geq 8$ \\
\hline \multirow[t]{6}{*}{ KPC } & + & KPC & + & Imipenem & $\geq 16$ \\
\hline & & & & Cefoxitin & $\geq 64$ \\
\hline & & & & Cefatriaxone & $\geq 64$ \\
\hline & & & & Amikacin & $\geq 64$ \\
\hline & & & & $\begin{array}{l}\text { Amoxicillin/clavulanate } \\
\text { potassium }\end{array}$ & $\geq 32$ \\
\hline & & & & Cefuroxime sodium & $\geq 64$ \\
\hline
\end{tabular}

Note: *Antibacterial circle diameter.

Abbreviations: MIC, minimum inhibitory concentration; KPC, Klebsiella pneumoniae carbapenemase.

subsequent CSF cultures were positive for XDR K. pneumoniae. On the 12th day of hospitalization, the original bilateral EVD tube implanted in the local hospital was removed because of frequent obstruction, and a new one was implanted in the right ventricle. However, the EVD tube was frequently obstructed, and a cranial CT scan revealed separation of the ventricles (Figure 3A and B). Given that polymyxin could not be administered intracerebroventricularly to achieve an effective concentration, the antibiotic regimen was adjusted to $2.5 \mathrm{~g}$ of CAZ/AVI IV at $8 \mathrm{~h}$ intervals and $400 \mathrm{mg}$ of amikacin IV once daily. After 6 days (19th day of hospitalization), CSF culture was first negative, and the 
Table 2 CSF Analyses of Case I on Different Date

\begin{tabular}{|c|c|c|c|c|c|}
\hline Day & $\begin{array}{l}\text { CSF RBC Count } \\
\text { (Cells/mm3) }\end{array}$ & $\begin{array}{l}\text { CSF WBC Count } \\
(\text { Cells } / \mathrm{mm} 3)\end{array}$ & $\begin{array}{l}\text { CSF \% } \\
\text { Neutrophil }\end{array}$ & $\begin{array}{l}\text { CSF Glucose } \\
\text { (g/L) }\end{array}$ & $\begin{array}{l}\text { CSF Protein } \\
(\mathrm{mmol} / \mathrm{L})\end{array}$ \\
\hline I & NA & NA & NA & 0.1 & 4.377 \\
\hline 3 & 100 & 4500 & 96 & 0.1 & 5.011 \\
\hline 5 & 30 & 800 & 60 & 2.0 & 3.095 \\
\hline 8 & 4480 & 60 & 46 & I. 4 & 3.997 \\
\hline 11 & 3000 & 15 & NA & 0.9 & 3.743 \\
\hline 12 & 1040 & 40 & 60 & 0.6 & 4.511 \\
\hline 14 & 250,000 & 9000 & 85 & 0.6 & 3.398 \\
\hline 15 & 2500 & 300 & 75 & I.I & 3.301 \\
\hline 16 & 7500 & 30 & 40 & 0.7 & 3.666 \\
\hline 18 & 1150 & 12 & NA & 1.0 & 3.293 \\
\hline 20 & 500 & 110 & 20 & 1.3 & 1.609 \\
\hline 21 & 100 & 8 & NA & 1.3 & 3.278 \\
\hline 23 & 10 & 90 & 20 & 2.2 & 1.768 \\
\hline 25 & 0 & 140 & 6 & 2.2 & 1.415 \\
\hline 31 & 20 & 20 & NA & 2.2 & 1.086 \\
\hline
\end{tabular}

Abbreviations: CSF, cerebrospinal fluid; RBC, red blood cell; WBC, white blood cell; NA, not available.

number of leucocytes and glucose levels in CSF gradually normalized. On the 40th day of hospitalization, antibiotic administration was discontinued after repeated negative results from CSF cultures. Then, the patient was transferred to a rehabilitation facility. The clinical course of treatment for Case 2 is summarized in Figure 4.

\section{Case 3}

A 21-year-old male was admitted to a local hospital for head trauma and successively underwent left decompressive craniectomy, external fixation of a pelvic fracture, internal fixation of a left femoral fracture, internal fixation of ankle repositioning, tracheotomy, and ventriculoperitoneal shunt. A month later, the patient was transferred to our hospital after being diagnosed with recurrent fever. CSF tests suggested acute septic meningitis (Table 6). The patient was empirically treated with $1.5 \mathrm{~g}$ of meropenem IV every $6 \mathrm{~h}$ and $600 \mathrm{mg}$ of linezolid IV every 12 $\mathrm{h}$. Given that the patient had an intracranial infection, ventricular terminal of the V-P shunt device was retained while the peritoneal terminal drainage tube was moved out of the body, making it an EVD. On the 10th day of hospitalization, CSF culture revealed carbapenem resistant MDR $P$. aeruginosa (Table 7). Antibiotic administrations
- Admission

- Debridement and EVD

- Meropenem $2 \mathrm{~g}$ q8h IV

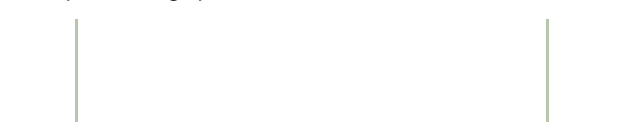

- CSF culture firstly negative
- Repeating negative results of CSF cultures negative

- Discontinuation of antibiotics

- Transformed to a rehabilitation facility

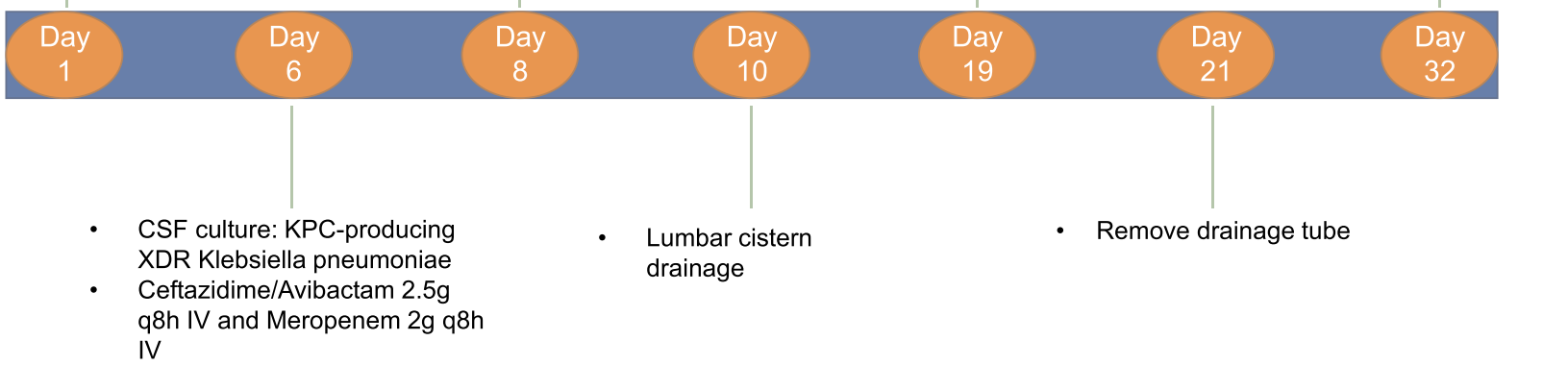

Figure 2 Summary of the clinical treatment process of Case I. 
Table 3 Susceptibility Results for Klebsiella pneumoniae in Cerebrospinal Fluid Collected on Different Date in case 2

\begin{tabular}{|c|c|c|c|}
\hline \multirow[t]{2}{*}{ Drug } & \multicolumn{3}{|c|}{ MIC (mg/L) } \\
\hline & $9 / 7$ & $9 / 10$ & $9 / 12$ \\
\hline Ceftazidime/avibactam & 4 & 4 & 4 \\
\hline Polymyxin & 1 & I & 1 \\
\hline Imipenem & $\geq 16$ & $\geq 16$ & $\geq 16$ \\
\hline Levofloxacin & $\geq 8$ & $\geq 8$ & $\geq 8$ \\
\hline Cefuroxime Sodium & $\geq 64$ & $\geq 64$ & $\geq 64$ \\
\hline Cefuroxime Axetil & $\geq 64$ & $\geq 64$ & $\geq 64$ \\
\hline Sulfamethoxazole & $\geq 320$ & $\geq 320$ & $\geq 320$ \\
\hline Ceftazidime & $\geq 64$ & $\geq 64$ & $\geq 64$ \\
\hline Cefepime & $\geq 32$ & $\geq 32$ & $\geq 32$ \\
\hline Piperacillin/tazobactam sodium & $\geq 128$ & $\geq 128$ & $\geq 128$ \\
\hline Cefoperazone/sulbactam & $\geq 64$ & $\geq 64$ & $\geq 64$ \\
\hline Tigecycline & 2 & 2 & 2 \\
\hline Ertapenem & $\geq 8$ & $\geq 8$ & $\geq 8$ \\
\hline Cefoxitin & $\geq 64$ & $\geq 64$ & $\geq 64$ \\
\hline Cefatriaxone & $\geq 64$ & $\geq 64$ & $\geq 64$ \\
\hline Amikacin & $\leq 2$ & $\leq 2$ & $\leq 2$ \\
\hline Amoxicillin/clavulanic acid & $\geq 32$ & $\geq 32$ & $\geq 32$ \\
\hline
\end{tabular}

Abbreviation: MIC, minimum inhibitory concentration.

were adjusted to 500,000 units of polymyxin IV every 8 $\mathrm{h}$ combined with intracerebroventricular injection of 50,000 units of polymyxin each day. The patient remained febrile, blood tests revealed leukocytosis, while sputum cultures revealed a MDR Pseudomonas aeruginosa infection. On the 13th day of hospitalization, antibiotic regimen were adjusted to 500,000 units of polymyxin IV every 8 $\mathrm{h}$ and $400 \mathrm{mg}$ of amikacin IV every $12 \mathrm{~h}$, combined with an intracerebroventricular injection of 50,000 units of polymyxin every day. After 3 days, urine cultures suggested a fungal infection, and fluconazole was also administered. Two weeks later (30th day of hospitalization), the original shunt was removed and EVD was performed for continued CSF drainage. With 24 days of treatment with intravenous and intraventricular polymyxin, CSF tests still revealed apparent leukocytosis with multiple positive CSF cultures of MDR Pseudomonas aeruginosa. Besides,
Table 4 Susceptibility Results for Pseudomonas aeruginosa in Sputum Collected on Different Date

\begin{tabular}{|l|l|l|l|l|}
\hline \multirow{2}{*}{ Drug } & \multicolumn{4}{|c|}{ MIC (mg/L) } \\
\cline { 2 - 5 } & Day 2 & Day 3 & Day 4 & Day 6 \\
\hline Ciprofloxacin & $\geq 4$ & $\geq 4$ & $\geq 4$ & $\geq 4$ \\
\hline Polymyxin & 4 & 4 & 4 & 4 \\
\hline Imipenem & $\geq 16$ & $\geq 16$ & $\geq 16$ & $\geq 16$ \\
\hline Cefoperazone/Sulbactam & $\geq 64$ & $\geq 64$ & $\geq 64$ & $\geq 64$ \\
\hline Ceftazidime & 32 & 32 & 32 & 32 \\
\hline $\begin{array}{l}\text { Ticarcillin/Potassium } \\
\text { Clavulanate }\end{array}$ & $\geq 128$ & $\geq 128$ & $\geq 128$ & $\geq 128$ \\
\hline Tobramycin & & & & \\
\hline Cefepime & $\leq 1$ & $\leq 1$ & $\leq 1$ & $\leq 1$ \\
\hline Meropenem & $\geq 32$ & $\geq 32$ & $\geq 32$ & $\geq 32$ \\
\hline Amikacin & $\geq 16$ & $\geq 16$ & $\geq 16$ & $\geq 16$ \\
\hline Levofloxacin & 4 & 4 & 4 & 4 \\
\hline
\end{tabular}

Abbreviation: MIC, minimum inhibitory concentration.

sputum and feces cultures suggested the presence of carbapenem-resistant $K$. pneumoniae (CRKP), which was sensitive to tigecycline and CAZ/AVI. Renal functions of the patient were significantly deteriorated. On the 34th day of hospitalization, polymyxin was discontinued since it can exacerbate renal impairments, and antibiotic administration was changed to $2.5 \mathrm{~g}$ of CAZ/AVI IV every $8 \mathrm{~h}$ and $600 \mathrm{mg}$ of amikacin IV every $12 \mathrm{~h}$. The CSF test normalized and the CSF culture was negative after 6 days of treatment with the new antibiotic regimen. Two weeks later (54th day of hospitalization), with repeated negative results of CSF cultures, the EVD tube was removed and antibiotic administration was discontinued. Then, the patient was subjected to cranioplasty as well as ventriculoperitoneal shunt implantation and transferred to a rehabilitation facility for further treatment. CSF and susceptibility test results for Pseudomonas aeruginosa are shown in Tables 6 and 7. A summary of the clinical treatment process of Case 3 is shown in Figure 5.

\section{Discussion}

MDR/XDR Gram-negative bacillus is a major global health threat. Treatment of infections associated with these bacteria has become increasingly challenging due to the lack of effective antibiotics. ${ }^{3}$ Treatment of MDR/XDR Gram- 
Table 5 CSF Analyses of Case 2 on Different Date

\begin{tabular}{|c|c|c|c|c|c|}
\hline Day & $\begin{array}{l}\text { CSF RBC Count } \\
(\text { Cells } / \mathrm{mm} 3)\end{array}$ & $\begin{array}{l}\text { CSF WBC Count } \\
(\text { Cells } / \mathrm{mm} 3)\end{array}$ & $\begin{array}{l}\text { CSF \% } \\
\text { Neutrophil }\end{array}$ & $\begin{array}{l}\text { CSF Glucose } \\
\text { (g/L) }\end{array}$ & $\begin{array}{l}\text { CSF Protein } \\
(\mathrm{mmol} / \mathrm{L})\end{array}$ \\
\hline 1 & 8250 & 1950 & 93 & 2.5 & 2.0 \\
\hline 2 & 2000 & 400 & 80 & 2.6 & 1.69 \\
\hline 4 & 3700 & 1900 & 92 & 4.0 & 2.51 \\
\hline 5 & 10,000 & 1700 & 90 & 4.9 & 2.01 \\
\hline 6 & 630 & 100 & 88 & 6.5 & 2.49 \\
\hline 7 & 200 & 3 & NA & 3.6 & 2.66 \\
\hline 8 & 30 & 2 & NA & 5.2 & 2.85 \\
\hline II & 270 & 20 & NA & 2.5 & 2.85 \\
\hline 12 & 700 & 200 & 70 & 2.5 & 3.32 \\
\hline 14 & 6000 & 380 & 88 & 4.4 & 12.01 \\
\hline 16 & 120 & 10 & NA & 2.6 & 9.86 \\
\hline 19 & 2000 & 0 & NA & 1.8 & 2.24 \\
\hline 21 & 300 & 30 & 70 & 3.2 & 30.08 \\
\hline 22 & 50 & 250 & 60 & 2.5 & 27.47 \\
\hline 26 & 150 & 220 & 80 & 8.4 & 22.92 \\
\hline 32 & 12,000 & 200 & 90 & 3.9 & 32.1 \\
\hline 37 & 70 & 8 & NA & 3.0 & 0.62 \\
\hline 39 & 1 & 3 & NA & 4.0 & 2.14 \\
\hline 42 & 750 & 6 & NA & 4.1 & 1.41 \\
\hline 50 & 33 & I & NA & 3.7 & 1.14 \\
\hline
\end{tabular}

Abbreviations: CSF, cerebrospinal fluid; RBC, red blood cell; WBC, white blood cell; NA, not available.

negative bacillus-associated CNS infections is more difficult due to inadequate penetration of antibiotics into the CNS.

Notably, CAZ/AVI is a combination antibiotic of cephalosporin and a $\beta$-lactamase inhibitor that has been approved for the treatment of complicated urinary tract infections, complicated intra-abdominal infections, and hospitalacquired pneumonia caused by Gram-negative bacteria. ${ }^{4}$
However, clinical studies on the efficacies of CAZ/AVI in the treatment of CNS infections are unavailable. Besides, clinical efficacies of CAZ/AVI for CNS infections are limited to case reports. Based on a PubMed search, only 6 adult cases of CNS infections caused by MDR/XDR K. pneumoniae $^{5-8}$ or $P$. aeruginosa ${ }^{8-10}$ were effectively treated using CAZ/AVI (Table 8).
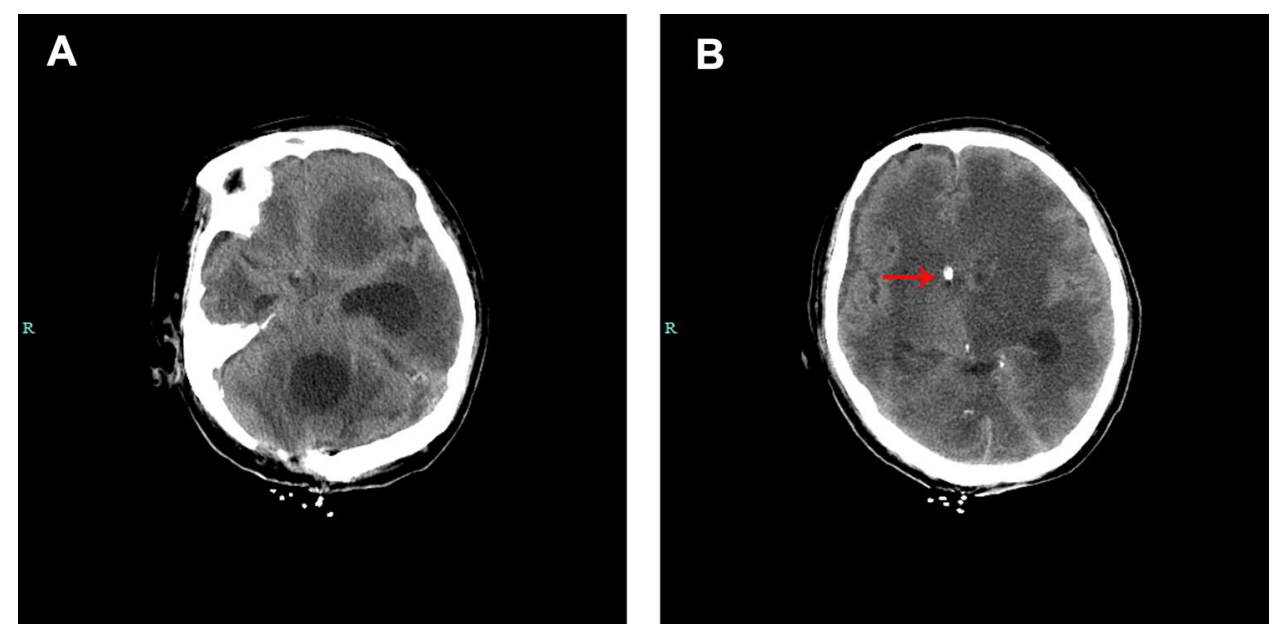

Figure 3 Brain computed tomography scan of case 2. (A) The CT images show blocking of the right lateral ventricle but with obvious enlargement of the left lateral ventricle and the fourth ventricle, which indicate separation of the ventricular system. (B) Red arrow show the drainage tube. 
Table 6 CSF Analyses of Case 3 on Different Date

\begin{tabular}{|c|c|c|c|c|c|}
\hline Day & $\begin{array}{l}\text { CSF RBC Count } \\
(\text { Cells } / \mathrm{mm} 3)\end{array}$ & $\begin{array}{l}\text { CSF WBC Count } \\
\left(\text { Cells } / \mathrm{mm}^{3}\right)\end{array}$ & $\begin{array}{l}\text { CSF \% } \\
\text { Neutrophil }\end{array}$ & $\begin{array}{l}\text { CSF Glucose } \\
\text { (g/L) }\end{array}$ & $\begin{array}{l}\text { CSF Protein } \\
(\mathrm{mmol} / \mathrm{L})\end{array}$ \\
\hline I & 8 & 9000 & 92 & 0.1 & 2.630 \\
\hline 3 & 40 & 1000 & 85 & 0.2 & 2.390 \\
\hline 6 & 5 & I & NA & 0.4 & 1.700 \\
\hline 8 & 44 & 20 & NA & 0.1 & 1.510 \\
\hline 11 & 45 & 113 & 10 & 0.0 & 2.660 \\
\hline 17 & 20 & 450 & 96 & 3.8 & 2.230 \\
\hline 18 & 4500 & 430 & 95 & 4.2 & 2.620 \\
\hline 19 & 4644 & 230 & 90 & 3.5 & 2.510 \\
\hline 20 & 40 & 15 & NA & 0.7 & 2.270 \\
\hline 24 & 1902 & 13,302 & 81 & 3.1 & 3.900 \\
\hline 25 & 90 & 15 & NA & 2.6 & 2.810 \\
\hline 28 & 3089 & 840 & 95 & 3.5 & 1.330 \\
\hline 29 & 240 & 212 & 95 & 3.3 & 1.160 \\
\hline 30 & 0 & 20 & NA & 3.4 & 1.730 \\
\hline 34 & 270 & 6 & NA & 3.6 & 0.990 \\
\hline 38 & 69 & 5 & NA & 2.6 & 1.070 \\
\hline 47 & 120 & 5 & NA & 3.2 & 0.520 \\
\hline 52 & 20 & 6 & NA & 3.0 & 0.510 \\
\hline 79 & 500 & 20 & NA & 2.8 & 0.280 \\
\hline
\end{tabular}

Abbreviations: CSF, cerebrospinal fluid; RBC, red blood cell; WBC, white blood cell; NA, not available.

We report 3 cases whose CNS infections were recovered through intravenous injections of CAZ/AVI combined with other antibiotics. In our first case, the CSF culture revealed a KPC-producing XDR K. pneumoniae infection. Based on susceptibility test results, the therapeutic option of CAZ/AVI combined with meropenem IV was used. With 13 days of such therapy and CSF drainage, the CSF culture became negative. Both ceftazidime and avibactam have been reported to have a high mean CSF penetration and the combination effectively suppresses CSF bacterial loads. ${ }^{4}$ The high CSF concentration of CAZ/AVI is vital for the treatment of CNS infections, ensuring a successful single intravenous therapy.

- CSF culture (local hospital): XDR Klebsiella pneumoniae

- Polymyxin 500000U IV $\mathrm{q} 12 \mathrm{~h}$ and tigecycline 100 $\mathrm{mg}$ IV q12h

- Polymyxin 500000 intracerebroventricularly qd

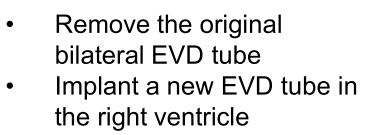

- Discontinuation of antibiotics

- Transformed to a rehabilitation facility
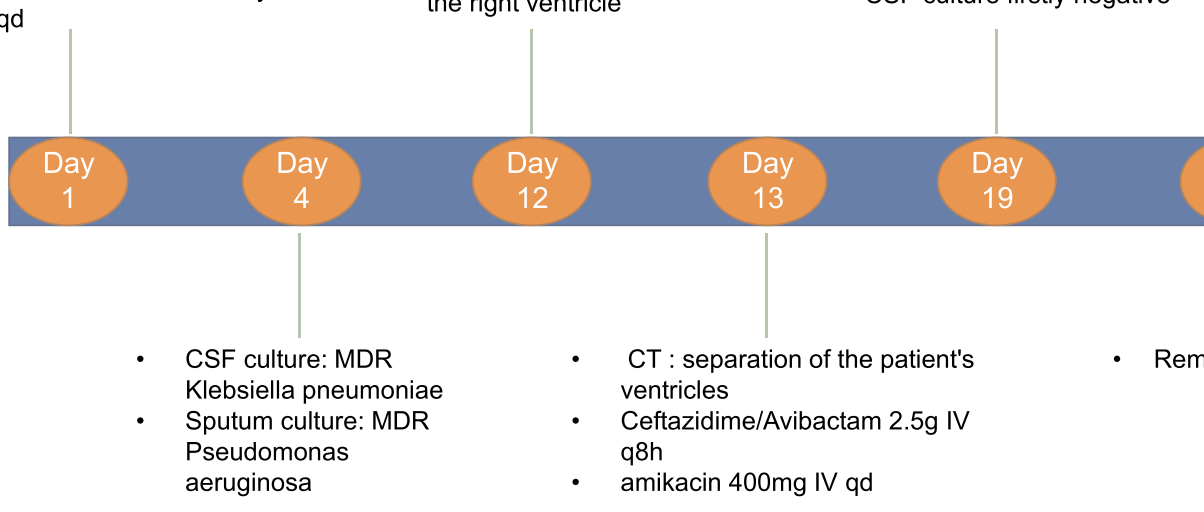

\begin{tabular}{c|cccc|} 
Day & Day & Day & Day & Day \\
12 & 13 & 19 & 20 & 40 \\
\hline
\end{tabular}

Figure 4 Schematic presentation of the clinical treatment process for Case 2. 
Table 7 Susceptibility Results for Pseudomonas aeruginosa in Cerebrospinal Fluid Collected on Different Date

\begin{tabular}{|l|l|l|l|}
\hline \multirow{2}{*}{ Drug } & \multicolumn{3}{|c|}{ MIC (mg/L) } \\
\cline { 2 - 4 } & Day I0 & Day I7 & Day 34 \\
\hline Ciprofloxacin & $\geq 4$ & $\geq 4$ & $\geq 4$ \\
\hline Polymyxin & $\leq 0.5$ & $\leq 0.5$ & $\leq 0.5$ \\
\hline Imipenem & $\geq 16$ & $\geq 16$ & $\geq 16$ \\
\hline Cefoperazone/Sulbactam & $\geq 64$ & 32 & $\geq 64$ \\
\hline Ceftazidime & 8 & 16 & 32 \\
\hline Ticarcillin/Potassium Clavulanate & $\geq 128$ & $\geq 128$ & $\geq 128$ \\
\hline Tobramycin & $\leq 1$ & $\leq 1$ & $\leq 1$ \\
\hline Cefepime & 16 & 16 & 16 \\
\hline Meropenem & $\geq 16$ & $\geq 16$ & $\geq 16$ \\
\hline Amikacin & $\leq 2$ & $\leq 2$ & 16 \\
\hline Levofloxacin & $\geq 8$ & $\geq 8$ & $\geq 8$ \\
\hline
\end{tabular}

Abbreviation: MIC, minimum inhibitory concentration.

The other two patients with CNS infections (one with carbapenem-resistant K. pneumoniae and one with MDR $P$. aeruginosa were initially treated with intravenous and intraventricular injections of polymyxin. As for the CNS infection caused by MDR/XDR Gram-negative bacillus, intravenous injections of sensitive antibiotics, primarily polymyxins and colistins, were ineffective because of the low penetration of the blood-brain barrier, particularly for severe CNS infections, including ventriculitis. Intraventricular/intrathecal injection of antibiotics is a vital therapy for CNS infections of such MDR/XDR Gram-negative bacillus, and sometimes a rescuing method. However, CNS infections caused by Gram-negative bacillus increase CSF protein concentrations, which obstruct CSF drainage. In case two, the EVD of the patient was often obstructed, making intraventricular injection difficult. Thus, we changed the therapy to intravenous CAZ/ AVI. Intraventricular injection of antibiotics aims to increase CSF concentrations; however, it is associated with various side effects, including chemical meningitis and epilepsy, which should be monitored. ${ }^{11}$ Despite intraventricular colistin and polymyxin being used in the treatment of Gram-negative ventriculitis and meningitis, IDSA guidelines indicate that intraventricular antimicrobials are not approved by the US Food and Drug Administration. Moreover, the evidence recommending their general use is insufficient. ${ }^{12}$ Besides, renal toxicity of colistin/polymyxin should be monitored. In case three, renal functions were significantly deteriorated, although renal dysfunction
- Craniocerebral trauma

- Ventriculoperitoneal shunt

- Tracheotomy

- Pelvic fracture

- Femoral fracture
- CSF culture: CRE Pseudomonas aeruginosa

- Polymyxin 500000U IV q8h

- Polymyxin 50000U intracerebroventricularly qd
- $\quad$ Urine culture suggests fungal infection

- Added Fluconazole

\section{Day} Day
7

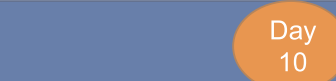

10

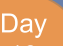

13

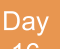

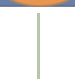

- Meropenem 1.5g IV q6h

- Remove the shunt

- External ventricular drainage and irrigation
- Linezolid 600mg IV q12h

- Pull out the peritoneal terminal drainage tube

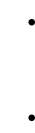

$$
\text { - }
$$

returned to normal

CSF culture was negative

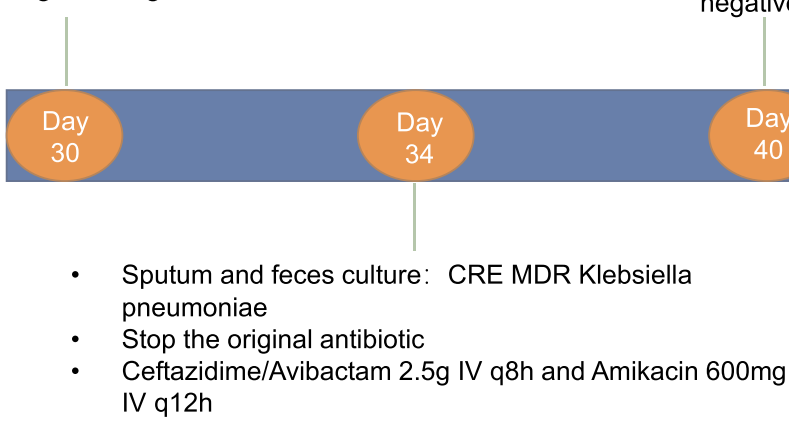

\section{Day}

40

Day

Added Amikacin 400mg IV q12h

- Cranioplasty

- Transfer to rehabilitation institution

Figure 5 Schematic presentation of the clinical treatment process for Case 3. 
Table 8 Summary of Ceftazidime-Avibactam Used for CNS Infections Caused by MDR K. pneumoniae and MDR P. aeruginosa

\begin{tabular}{|c|c|c|c|c|c|}
\hline $\begin{array}{l}\text { First } \\
\text { Author }\end{array}$ & Country & Numbers & CSF Cultures & Drugs & Outcome \\
\hline Holyk $^{7}$ & USA & I & MDR K. pneumoniae & $\begin{array}{l}\text { CAZ/AVI } 2.5 \mathrm{~g} \text { IV } 2 \mathrm{I} \text { days and gentamicin } 15 \\
\text { days intraventricular }\end{array}$ & Cured \\
\hline Samuel $^{5}$ & USA & I & KPC-producing $K$. pneumoniae & CAZ/AVI $2.5 \mathrm{~g}$ IV q6h I4 days & Cured \\
\hline Gofman $^{8}$ & USA & I & $\begin{array}{l}\text { Carbapenem-resistant } K \text {. pneumoniae and } \\
\text { Pseudomonas aeruginosa }\end{array}$ & $\begin{array}{l}\text { Intrathecal amikacin } 30 \mathrm{mg} \text { qd } 4 \text { weeks and } \\
\text { CAZ/AVI } 2.5 \mathrm{~g} \mathrm{IV} \mathrm{q8h} 6 \text { weeks }\end{array}$ & Cured \\
\hline Yasmin $^{6}$ & UAS & I & KPC-producing MDR K. pneumoniae & CAZ/AVI $2.5 \mathrm{~g} I V$ q8h 10 days & Cured \\
\hline Xipell ${ }^{9}$ & Spain & I & XDR Pseudomonas aeruginosa & $\begin{array}{l}\text { CAZ/AVI } 2.5 \mathrm{~g} \text { and colistin } 2 \mathrm{MU} \text { IV q8h } 30 \\
\text { days }\end{array}$ & Cured \\
\hline $\begin{array}{l}\text { Rodríguez- } \\
\text { Núñez }\end{array}$ & Spain & I & XDR Pseudomonas aeruginosa & CAZ/AVI 3I days & $\begin{array}{l}\text { Alive at } 90 \\
\text { days }\end{array}$ \\
\hline
\end{tabular}

Abbreviations: CSF, cerebrospinal fluid; KPC, Klebsiella pneumoniae carbapenemase; MDR, multidrug-resistant; XDR, extensively drug-resistant; CAZ/AVI, ceftazidime/ avibactam; IV, intravenously.

might not be as a result of polymyxin. However, since polymyxin might exacerbate renal impairment, we changed the therapy to intravenous CAZ/AVI. Among the two patients, intravenous injections of CAZ/AVI combined with amikacin were performed after the failure or onset of suspected side effects of polymyxin (combined intravenous and intraventricular injection), and effectively treated infections.

Conclusive evidence for CAZ/AVI in combination with other antibiotics for the treatment of MDR Gram-negative bacteria associated meningitis remain unreported. Xipell et al reported that CAZ/AVI combined with other antibiotics synergizes and prevents selective resistance mutations in bacteria, although this is not reliably supported by evidence. ${ }^{9}$ Among the six cases reported in previous studies, three cases were treated with intravenous CAZ/AVI alone, while three cases were treated with CAZ/AVI IV combined with another antibiotic for CNS infections, including ventricular injection of gentamicin, intrathecal injection of amikacin, and intravenous injection of colistin. Among the three cases, CAZ/AVI was used in combination with other antibiotics. In the first case, antibiotic susceptibility revealed a synergistic effect of CAZ/AVI and meropenem in treating KPC-producing XDR Klebsiella pneumoniae infection. Similarly, Gaibani et al reported that the combination of CAZ/AVI and meropenem exhibited synergism against KPC-Kp isolates. ${ }^{13}$ These findings suggest that CAZ/AVI inhibits the effects of KPC and enhances the anti-infectious effect of meropenem. Amikacin is an antibiotic that is frequently used in combination with CAZ/AVI to treat MDR/XDR Gramnegative bacillus infection. ${ }^{14-16}$ In the second and third cases, we used CAZ/AVI IV combined with amikacin to effectively treat the CNS infections.

The optimal treatment duration for a post-neurosurgical CNS infection with MDR and XDR Gram-negative bacilli has not been established. Infectious Diseases Society of America (IDSA) guidelines recommend 10-14 days of treatment for Gram-negative bacilli; however, the duration of therapy needs to be individualized based on clinical responses of the patient. ${ }^{12} \mathrm{We}$ administered the antibiotics until the CSF of the patients normalized and at least $3 \mathrm{CSF}$ cultures (interval of sampling $>$ one day) were negative. None of the three patients had a recurrence of CNS infection during subsequent follow-up visits.

In addition to antibiotics, timely surgical intervention is vital. Based on IDSA guidelines, removal of the infected shunt or extracerebral ventricular drain, reasonable extracerebral drainage, combined with appropriate antimicrobial therapy, is arguably the most effective treatment for cerebrospinal fluid catheter infections. ${ }^{12}$ In our cases, CSF drainage (EVD or lumbar cistern drainage) and prompt removal of the abscess were important and the basis for antibiotic treatment.

There are several limitations to the use of CAZ/AVI in CNS infections caused by MDR/XDR Gram-negative bacillus. First, the number of studies evaluating the toxicity of CAZ/AVI on CNS directly are limited. Second, we used 2.5 $\mathrm{g}$ of CAZ/AVI intravenously every $8 \mathrm{~h}$ to treat CNS infections caused by MDR/XDR Gram-negative bacillus as reported in literature. However, it has not been established whether this 
dose is associated with other side effects in brain tissues as well as in patients. Due to the limited number of patients in this case study, we did not find any other adverse events in the three patients. In addition, we are uncertain if intravenous CAZ/AVI is safe and effective at other doses for the treatment of CNS infections that are associated with MDR/XDR Gramnegative bacteria. This should be further investigated.

\section{Conclusion}

We described 3 patients with MDR/XDR Gram-negative bacillus-associated CNS infections that were treated with CAZ/AVI. Based on the reported cases in this study and from literature, intravenous administration of CAZ/AVI in combination with other antibiotics might be a valuable approach for the treatment of CNS infections due to MDR/XDR Gram-negative bacteria and a key supplement to the intraventricular injection of colistin/polymyxin. However, further studies and clinical trials are necessary to establish the efficacies of CAZ/AVI in CNS infections.

\section{Data Sharing Statement}

All data generated during this study are included in the tables.

\section{Ethics Approval and Informed Consent}

The study was approved by the Ethics Committee of The First Affiliated Hospital, College of Medicine, Zhejiang University, Hangzhou, China.

\section{Consent for Publication}

The next of kin of patients have provided informed consent for publication of this Case report and accompanying images and video.

\section{Acknowledgments}

Thanks to Freescience for the linguistic editing of the article. Qian Zhou, Hao Wang: These authors are co-first authors.

\section{Author Contributions}

All authors made substantial contributions to conception and design, acquisition of data, or analysis and interpretation of data; took part in drafting the article or revising it critically for important intellectual content; agreed to submit to the current journal; gave final approval of the version to be published; and agree to be accountable for all aspects of the work.

\section{Funding}

This project was supported by The National Natural Science Foundation of China (No. 81971159 and No. 81470052). These funds support the literature download for this study.

\section{Disclosure}

The authors declare no competing interests.

\section{References}

1. van de Beek D, Brouwer MC, Thwaites GE, Tunkel AR. Advances in treatment of bacterial meningitis. Lancet. 2012;380 (9854):1693-1702. doi:10.1016/s0140-6736(12)61186-6

2. Barber KE, Ortwine JK, Akins RL. Ceftazidime/avibactam: who says you can't teach an old drug new tricks? J Pharm Pharm Sci. 2016;19 (4):448-464. doi: $10.18433 / \mathrm{j} 3 \times 31 \mathrm{r}$

3. Karaiskos I, Giamarellou H. Multidrug-resistant and extensively drug-resistant Gram-negative pathogens: current and emerging therapeutic approaches. Expert Opin Pharmacother. 2014;15 (10):1351-1370. doi:10.1517/14656566.2014.914172

4. Shirley M. Ceftazidime-avibactam: a review in the treatment of serious Gram-negative bacterial infections. Drugs. 2018;78 (6):675-692. doi:10.1007/s40265-018-0902-x

5. Samuel S, Edwards NJ, Rojas LJ, et al. Ceftazidime-avibactam for the treatment of post-neurosurgical meningitis caused by a KPC-producing Klebsiella pneumoniae. Open Forum Infect Dis. 2016;3(suppl_1).

6. Yasmin M, Hanrahan J, Marshall S, et al. Using therapeutic drug monitoring to treat KPC-producing Klebsiella pneumoniae central nervous system infection with ceftazidime/avibactam. Open Forum Infect Dis. 2020;7(9):ofaa349. doi:10.1093/ofid/ofaa349

7. Holyk A, Belden V, Lee JJ, et al. Ceftazidime/avibactam use for carbapenem-resistant Klebsiella pneumoniae meningitis: a case report. J Antimicrob Chemother. 2018;73(1):254-256. doi:10.1093/ $\mathrm{jac} / \mathrm{dkx} 358$

8. Gofman N, To K, Whitman M, Garcia-Morales E. Successful treatment of ventriculitis caused by Pseudomonas aeruginosa and carbapenem-resistant Klebsiella pneumoniae with i.v. ceftazidime-avibactam and intrathecal amikacin. Am J Health-Syst Pharm. 2018;75(13):953-957. doi:10.2146/ajhp170632

9. Xipell M, Bodro M, Marco F, Losno RA, Cardozo C, Soriano A. Clinical experience with ceftazidime/avibactam in patients with severe infections, including meningitis and lung abscesses, caused by extensively drug-resistant Pseudomonas aeruginosa. Int J Antimicrob Agents. 2017;49(2):266-268. doi:10.1016/j.ijantimicag.2016.11.005

10. Rodríguez-Núñez O, Ripa M, Morata L, et al. Evaluation of ceftazidime/avibactam for serious infections due to multidrug-resistant and extensively drug-resistant Pseudomonas aeruginosa. $J$ Glob Antimicrob Resist. 2018;15:136-139. doi:10.1016/j.jgar.2018.07.010

11. Lauretti L, D'Alessandris QG, Fantoni M, et al. First reported case of intraventricular tigecycline for meningitis from extremely drug-resistant Acinetobacter baumannii. J Neurosurg. 2017;127 (2):370-373. doi:10.3171/2016.6.jns16352

12. Tunkel AR, Hasbun R, Bhimraj A, et al. 2017 Infectious Diseases Society of America's Clinical Practice Guidelines for HealthcareAssociated Ventriculitis and Meningitis. Clin Infect Dis. 2017;64(6): e34-e65. doi:10.1093/cid/ciw861

13. Gaibani P, Lewis RE, Volpe SL, et al. In vitro interaction of ceftazidime-avibactam in combination with different antimicrobials against KPC-producing Klebsiella pneumoniae clinical isolates. Int J Infect Dis. 2017;65:1-3. doi:10.1016/j.ijid.2017.09.017 
14. Mikhail S, Singh NB, Kebriaei R, et al. Evaluation of the synergy of ceftazidime-avibactam in combination with meropenem, amikacin, aztreonam, colistin, or fosfomycin against well-characterized multidrug-resistant Klebsiella pneumoniae and pseudomonas aeruginosa. Antimicrob Agents Chemother. 2019;63(8). doi:10.1128/ aac.00779-19

15. Chen T, Xu W, Yu K, et al. In vitro activity of ceftazidime-avibactam alone and in combination with amikacin against colistin-Resistant Gram-negative pathogens. Microb Drug Resist. 2020. doi:10.1089/ mdr.2019.0463
16. Almarzoky Abuhussain SS, Kuti JL, Nicolau DP. Antibacterial activity of human simulated epithelial lining fluid concentrations of ceftazidime-avibactam alone or in combination with amikacin inhale (BAY41-6551) against carbapenem-resistant pseudomonas aeruginosa and Klebsiella pneumoniae. Antimicrob Agents Chemother. 2018;62(7). doi:10.1128/aac.00113-18

\section{Publish your work in this journal}

Infection and Drug Resistance is an international, peer-reviewed openaccess journal that focuses on the optimal treatment of infection (bacterial, fungal and viral) and the development and institution of preventive strategies to minimize the development and spread of resistance. The journal is specifically concerned with the epidemiology of antibiotic resistance and the mechanisms of resistance development and diffusion in both hospitals and the community. The manuscript management system is completely online and includes a very quick and fair peerreview system, which is all easy to use. Visit http://www.dovepress.com/ testimonials.php to read real quotes from published authors. 